\title{
Monitoring AI Services for Misuse
}

\author{
Seyyed Ahmad Javadi, Chris Norval, Richard Cloete, Jatinder Singh \\ Compliant \& Accountable Systems Group, Dept. of Computer Science \& Technology \\ University of Cambridge, UK \\ firstname(s).lastname@cst.cam.ac.uk
}

\begin{abstract}
Given the surge in interest in AI, we now see the emergence of Artificial Intelligence as a Service (AIaaS). AIaaS entails service providers offering remote access to ML models and capabilities at 'arms-length', through networked APIs. Such services will grow in popularity, as they enable access to state-of-the-art ML capabilities, 'on demand', 'out of the box', at low cost and without requiring training data or ML expertise.

However, there is much public concern regarding AI. AIaaS raises particular considerations, given there is much potential for such services to be used to underpin and drive problematic, inappropriate, undesirable, controversial, or possibly even illegal applications.

A key way forward is through service providers monitoring their AI services to identify potential situations of problematic use. Towards this, we elaborate the potential for 'misuse indicators' as a mechanism for uncovering patterns of usage behaviour warranting consideration or further investigation. We introduce a taxonomy for describing these indicators and their contextual considerations, and use exemplars to demonstrate the feasibility analysing AIaaS usage to highlight situations of possible concern. We also seek to draw more attention to AI services and the issues they raise, given AIaaS' increasing prominence, and the general calls for the more responsible and accountable use of AI.
\end{abstract}

\section{CCS CONCEPTS}

- Computing methodologies $\rightarrow$ Artificial intelligence; $•$ Computer systems organization $\rightarrow$ Cloud computing; $\bullet$ Social and professional topics $\rightarrow$ Computing / technology policy; • Security and privacy $\rightarrow$ Domain-specific security and privacy architectures.

\section{KEYWORDS}

artificial intelligence; machine learning; cloud; law; accountability; misuse; monitoring; audit; compliance; FAT; fairness; accountability; transparency MLaaS; AIaaS

ACM Reference Format:

Seyyed Ahmad Javadi, Chris Norval, Richard Cloete, Jatinder Singh. 2021. Monitoring AI Services for Misuse. In Proceedings of the 2021 AAAI/ACM Conference on AI, Ethics, and Society (AIES '21), May 19-21, 2021, Virtual Event, USA. ACM, New York, NY, USA, 11 pages. https://doi.org/10.1145/ 3461702.3462566

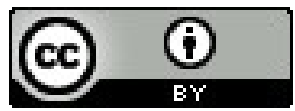

This work is licensed under a Creative Commons Attribution International 4.0 License. AIES '21, May 19-21, 2021, Virtual Event, USA. (C) 2021 Copyright held by the owner/author(s). ACM ISBN 978-1-4503-8473-5/21/05.

https://doi.org/10.1145/3461702.3462566

\section{INTRODUCTION}

There is a surge of interest in machine learning (ML), which is envisaged to transform a wide range of industries. ML, however, poses practical challenges, given that undertaking ML generally requires access to expertise, compute resources, and often significant amounts of data [36].

As such, we see the emergence of what is termed 'AI as a Service' (AIaaS). Offered by a range of organisations, most predominately the major cloud service providers, it attempts to meet the growing demands by providing ML capabilities 'out of the box' - such that customers (service users) can easily integrate ML functionality into their applications without having to undertake ML themselves. That is, AIaaS entails the on-demand provision of ML models and related services, whereby customers can send data (as inputs) through network APIs, receiving back the results of ML processes (predictions, classifications, decisions, etc). AIaaS offerings are often generic (some are customisable), and include services like text to speech, object detection, face recognition, text translation, etc.

At the same time, technology and its operators are increasingly the subject of public scrutiny. A series of problematic and controversial AI practices and uses have drawn criticism [7, 27], contributing to increasing demands for technologies to be more transparent, accountable, and legally compliant - as discussions of 'ethical AI', 'algorithmic accountability', and 'tech regulation' all demonstrate (e.g. see $[6,39,48])$.

Given AIaaS makes sophisticated, complex, and generalisable functionality readily and widely-accessible, often with little direct provider oversight, it stands that there is much scope for AIaaS services to be used to underpin or otherwise support controversial and problematic applications [26]. One can readily envisage, e.g., face and other image recognition services supporting inappropriate surveillance.

Providers have a clear interest in ensuring that their AIaaS services are used in appropriate, legal, and socially acceptable ways [26]. One driver is reputation; providers have an interest to not be seen as supporting problematic applications - a controversial application being branded as 'Powered by [Provider_X]' could lead to public backlash and undesirable attention. Indeed, as we later discuss, we have already seen instances of this, where some major providers have restricted the use of their face-related AIaaS services for law enforcement purposes [20]. Moreover, the legal responsibilities and liabilities of AIaaS providers are still unclear [8], while emerging regulatory regimes, such as the EU's Digital Services Act [13] and Artificial Intelligence Act [14] proposals, look set to force major providers to take more responsibility for how their platforms are used, and what they support.

This paper explores how providers might be more proactive in ensuring their AI services are being used appropriately, focusing on technical approaches for supporting this. We build and expand 
on our previous work [26]-which made the general, conceptual case for misuse monitoring-and show practical ways forward.

Specifically, we first re-introduce and elaborate the concept of misuse indicators: mechanisms that involve monitoring and analysing AIaaS usage patterns to bring providers' attention to certain happenings (regarding their customers' behaviour) on their platforms. As concerns are context-dependent, indicators would be deployed by providers as appropriate for the situation. Importantly, the purpose of the indicators is indeed to indicate - to highlight situations warranting consideration, rather than definitively describing misuse (though they might in some instances). That is, an indicator serves as a support mechanism to support follow-on actions, e.g. to direct attention to certain occurrences (like a warning system), provide evidence to support investigation, and so on.

Naturally, what can and should be analysed by such indicators is contextual, depending on various factors, such as the nature of the service, data sensitivity, the scale of the analysis required, etc. Towards this, we introduce a taxonomy of considerations, serving as a framework to assist the formulation of appropriate misuse indicators. We then consider misuse indicators 'in practice', through two exemplar scenarios which serve to illustrate how providers might navigate the usage landscape to highlight and uncover situations warranting attention. ${ }^{1}$ Importantly, our exemplars are only illustrative of how a provider might explore happenings on their platform. Our aim is to demonstrate the feasibility and potential for misuse indicators - what a misuse indicator looks like in practice will depend on the needs of the particular provider, their resources, and the relevant legal frameworks. We conclude with a brief discussion of broader issues, including issues of incentives, risks and power.

In all, our contributions are to (i) elaborate the issues of AIaaS misuse and the role for misuse monitoring; (ii) introduce and provide a taxonomy describing various considerations in defining misuse indicators; (iii) illustrate the feasibility and practical considerations regarding AI service monitoring through the use of exemplars; and (iv) discuss the broader implications of misuse monitoring and highlight interdisciplinary research opportunities in this space. Our broader aim is to draw urgent community attention to AIaaS and its implications for the responsible and accountable use of AI.

\section{AIaaS: EMERGENCE AND RISKS}

ML has gained considerable interest in recent years, and looks set to continue to pervade many aspects of everyday life [45].

ML is driven by data; it typically works to identify patterns and derive mathematical models from the underlying (input) data on which it is applied [11]. These models provide knowledge and insights, and can be applied to new data to output predictions, decisions, or classifications [25].

However, building ML models can be challenging. Not only does it require knowledge and expertise (said to be in short supply [50]), but such tasks typically require sufficient quantities of data, as well as substantial resources (compute), to build a model which is

\footnotetext{
${ }^{1}$ The exemplars are general, of a type to help providers understand and explore the service usage landscape. As opposed to those more specific, these types of indicators are a first step for uncovering situations of concern, providing the information to support the development of more narrow and definitive indicators targetting specific instances of misuse.
}

accurate and effective [22, 36]. Such requirements present a barrier for entry; not all those that seek to leverage ML will have the time, data, resources, or expertise to do so.

The result has been a burgeoning AI as a Service (AIaaS) market. AIaaS providers seek to make ML accessible, by providing customers with ML capabilities on-demand, 'out of the box', with "no machine learning skills required" [2].

\subsection{AIaaS Overview}

The most prominent AIaaS providers are the tech giants, the very companies with significant access to data, infrastructure and expertise required for effective ML [26].

AIaaS services can be broadly categorised as providing their customers (i) access to pre-built models and related services; (ii) access to hybrid or partially-trained models for customisation; or (iii) the supporting infrastructure for customers to undertake their own machine learning (e.g. ML frameworks, GPUs, etc). We consider the first and most common AIaaS category-access to pre-built models-though our work is also relevant for the other categories.

There are a range of pre-built models currently available through AIaaS providers; including models for object detection, speech transcription, facial recognition, and speech synthesis [2, 17, 24, 30], to name a few. These are accessible to customers through APIs: here, web-based interfaces through which the model-based services are used. Generally, a customer uses a service through its API, by sending to the provider a request specifying the particular service (e.g. object recognition, speech-to-text), and the data to be treated as inputs (e.g. an image, or audio file). After various checks (security, budget, etc), the provider will process the request and return the results of applying the AI service as a response [26].

In this way, AIaaS effectively provides a set of 'application building blocks', enabling customers to easily incorporate AI capabilities into their systems.

\subsection{The potential for misuse}

While the wider risks of AI have gained recent widespread attention [7], there has been considerably less focus on AI when offered as a service. AIaaS does, however, warrant consideration.

First, AI services represent powerful and scalable capabilities that drive applications $[8,26]$. They are becoming widely accessible; marketed as "ML with no experience required" shows they can be used by potentially anyone, without requiring the data, nor detailed knowledge, skills, or expertise otherwise required to undertake ML. It is therefore also likely that many AIaaS customers will have limited exposure to ML's wider considerations (ethics, bias, etc.).

Moreover, AI services are typically provisioned at 'a few clicks', often with little direct provider interaction, at low cost (pay per use). This low barrier to entry, ease of integration and use, limited provider involvement during engagement, the generalisability of such services across a wide range of contexts-indeed, all of which are AIaaS selling-points-means there is a real propensity for such services to support distasteful, problematic, controversial, or even illegal applications.

For some AIaaS services, e.g. facial recognition, the risks may be obvious [49]; indeed, several AIaaS providers responded to public concerns by stating their face recognition services must not be used 
for law enforcement [20]. Though even more seemingly innocuous services have the potential for problematic use; e.g. speech generation for impersonation [19, 46], speech-to-text in surveillance [33, 47], object recognition for tracking movements $[5,32]$, and various tools that ultimately support producing 'deepfakes' [21]. Some customers will be aware their service usage is problematic, and others won't. And issues may arise where a model intended for certain usage contexts is used elsewhere [41], e.g., due to representation biases.

Regardless of whether inadvertent or deliberate, inappropriate, controversial, and nefarious AI uses and applications (see, e.g., [44]) will continue to emerge. And as AIaaS provides easy access to ML capabilities, there is real potential for AI services to drive some of these problematic applications. There are clear benefits to understanding more about the types of abuse that might occur, and how they could be detected. Through doing so, steps can be identified and taken to detect and prevent the misuse of AIaaS.

\subsection{Drivers for tackling misuse}

There are a number of drivers that encourage providers to consider the implications of AI services.

Providers have already shown an interest in engaging in discussions of 'responsible' and 'accountable' AI; this to aid public acceptance of the technology they purvey and to avoid any general public backlash. For example, Google states that AI should protect privacy and be socially beneficial, fair, safe, and accountable to people; IBM discusses issues of trust and transparency [37]; and the Partnership on AI, a non-profit initiative (some of its lead organisations the major AIaaS providers) seeks to "better understand AI's impacts" [43].

Moreover, it is in the interest of AIaaS providers to ensure that their services are not being misused [26]. As discussed, this can be for reasons of reputation, to avoid any losses from being associated with an unsavoury application. Indeed, the public stance on facial recognition services for law enforcement is evidence that the providers take reputational concerns seriously [18], as is the fact that Microsoft Face rate limits the calls to their face API [31], while Amazon limits the number of faces that can be identified from an image [3].

Another driver is the provider's legal position regarding their obligations, liabilities and responsibilities for the AI services they offer, and how customers might use them. Currently, the legal position of providers, and whether and to what extent they are responsible for the applications their platforms enable, is rather unclear and requires further attention [8]. Interesting questions are raised given that AI services entail providers playing a crucial role in delivering actual application functionality (through model providing, predictions, decisions, etc) as opposed to more generic support infrastructure-oriented offerings of traditional cloud (or where the provider 'owns' the whole application as in SaaS) [8]. There are emerging legal and regulatory regimes relevant here, resulting from the increasing critical public discourse regarding AI (and tech generally). That is, beyond the ramp up of such with data protection regulations (e.g. GDPR, CCPA), we see emerging regulations, such as the EU's Digital Services Act [13] and Artificial Intelligence Act [14] proposals, which seek to place more direct obligations on service providers so that they are more responsible and accountable for the actions and outcomes their platforms enable. These look set to have direct implications for AIaaS providers.

Therefore, not only do organisations have a clear interest in ensuring that what they provide is used responsibly, but there will be increasing pressures for them to ensure that they do so. The concerns are socio-technical; providers will need high-level governance measures, like contractual obligations or terms of service. However, technical means (our focus here) also have a clear role, by placing 'eyes-on-the-ground' which helps assist providers in uncovering situations of problematic service use.

\section{AIaaS MISUSE DISCOVERY}

We now introduce the concept of misuse indicators: measures that analyse service usage patterns to help reveal to providers situations warranting attention.

\subsection{Misuse indicators}

AIaaS platforms already generate (log) much information about their service usage [42]. This information can serve a variety of purposes, e.g. to improve service performance, understand why a particular event happened (e.g. a crash/failure, where something 'unexpected' occurred), meeting legal obligations, and, of course, support billing (providers often charge per use transaction). Some AIaaS services also use the input data sent to their services in helping to further enhance and improve their ML models (e.g. [4]). This audit data can help in detecting misuse, by describing what a tenant did, when and how.

It follows that methods for monitoring the usage patterns and behaviours of customers can work to indicate and expose situations that might be suspicious, extraordinary, uncommon, or otherwise worthy of attention (or indeed, that particular usage or behaviours are not noteworthy). Providers can then take the appropriate actions, be they automated (alerts, reconfiguration such as changing $\log$ levels to provide more evidence, limiting service rates), or manual (i.e. investigations).

Tackling issues of misuse requires an understanding how services are being used. Towards this, the concept of a misuse indicator [26] refers to those undertakings that reveal to and alert a provider of certain happenings deemed of interest. The purpose is to support a provider in uncovering (and dealing with) situations in which their services are being misused by their customers. Indicators entail monitoring; the information feeding an indicator comes from audit data, i.e. data relating to the transactions an AIaaS customer makes with a service, and might relate to request timing, frequency, perhaps within and across services, and potentially the nature of the inputs and outputs as well.

In this way, misuse indicators work to inform of relevant occurrences regarding the nature of service use, which can help providers understand, categorise, identify, and prevent further misuse from occurring. The purpose is to uncover situations that warrant attention, which may or may not be situations of actual misuse. That is, misuse indicators might work to identify known situations of concern, e.g. where a face recognition service is being used in a particular way. Other indicators may be more exploratory-e.g. to identify anomalous or outlier behaviours, or simply to help 
profile and landscape the nature of the service usage (as we later explore)-highlighting the new and emerging patterns or trends possibly requiring investigation. Behaviour comparisons might be intra-customer, e.g. where a customer exhibits known problematic patterns of use, or where their behaviours suddenly change; or might be across customers, comparing differences in use, where, e.g., outliers may be of particular interest.

Naturally, the specifics of any indicator are contextual, depending on the provider's priorities and concerns, the service(s) in question, the risk profile/severity of harm, the intrusiveness of monitoring, among other factors. As such, after highlighting related work, we present a taxonomy of indicator considerations designed to help AIaaS providers take a more informed approach to mitigating service misuse.

\subsection{Monitoring behaviours in other domains}

Little attention has been given to misuse discovery in AIaaS, let alone practical methods for such.

However, analysing behavioural patterns has been considered in other domains, e.g. finance, security, and systems management (see $[1,9,16]$ respectively for surveys of approaches taken in these domains).

Naturally, the specifics of the methods employed are highly context-specific. Broadly speaking, however, there are two key classes of approach, which give a general flavour of the methods employed. Signature based approaches involve a pattern (i.e. a signature) that corresponds to a known situation. These involve comparing these known patterns against observed behaviours in order to detect particular situations of interest [28], and might entail, for example, a malicious string in a network packet, a particular spending pattern (e.g. two different locations at once), and so forth. Anomaly-based approaches involve building behaviour profiles to detect suspicious situations, often looking for outlier or deviations in behavioural patterns [28]. Machine learning methods can be employed [16], for instance to group behaviour patterns to identify common and outlier/anomalous behaviours, or to train classifiers that can be used to detect particular problematic situations. Signature- and anomaly-based methods, as well as the specifics of approaches employed in various other domains, have real potential to inspire (as we were) ways forward in developing and implementing AIaaS misuse indicators.

\section{AIaaS MISUSE INDICATORS: A TAXONOMY}

There are a range of possible AIaaS misuse indicators that might be employed. We now introduce a taxonomy to help formulate the development of misuse indicators. This taxonomy provides a lens for elaborating aspects relevant to an indicator's construction, and the broader considerations and implications of its deployment.

Table 1 represents the taxonomy, broken down into domains. The first domain relates to the source information driving the indicator. Within this, access level relates to the level of detail; that is, whether the indicator involves any metadata around the transaction (i.e. general information about the transaction such as its timestamp, customer-id), and/or if it concerns actual content of the request/response (I/O), which may be textual, numeric, images, sound, text, etc. The sensitivity attribute refers to the level of risk

\begin{tabular}{|c|c|c|}
\hline Domains & Dimension & Considerations \\
\hline \multirow{3}{*}{ Source Information } & \multirow{2}{*}{ Access level } & Metadata \\
\hline & & Content \\
\hline & Sensitivity & Sensitivity \\
\hline \multirow{8}{*}{ Misuse analysis } & \multirow{2}{*}{ Analysis type } & Trait-based \\
\hline & & Discovery-based \\
\hline & \multirow{3}{*}{ Scale } & Overheads \\
\hline & & Tenant-specific \\
\hline & & Across-tenants \\
\hline & \multirow{3}{*}{ Robustness } & Efficacy \\
\hline & & Representativeness \\
\hline & & Circumvention \\
\hline \multirow{3}{*}{ Record keeping } & \multirow{2}{*}{ Duration } & Temporary \\
\hline & & Permanent \\
\hline & Sensitivity & Sensitivity \\
\hline
\end{tabular}

Table 1: Taxonomy of misuse indicator considerations.

associated for the relevant information; i.e. does it refer to sensitive information regarding the customer or possible end-users (or others to which the data relates). This attribute also encapsulates any relevant legal obligations and risk. Considerations include whether the data might be confidential from a business perspective, private or personal, or perhaps 'special category' under the GDPR [15] (i.e. that considered "highly-personal" like biometrics or race), and other legal considerations.

The second domain refers to the process of misuse analysis, covering the specifics of the analysis undertaken. One dimension refers to the analysis type. These are similar to, but broader than the general classes of approach from other domains, as just described. Here, an analysis might be trait-based; looking for a particular pattern of behaviour known to be problematic or otherwise requiring attention (e.g. certain usage patterns which might be evident of population surveillance). Alternatively, the approach may be more discovery-based, more to help explore the usage landscape as a means for uncovering situations of possible interest (e.g. to reveal potentially abnormal or noteworthy behaviours).

The scale dimension refers to whether the analysis focuses on only particular customer(s), or whether it is a comparative analysis across a wide range of customers. It also entails considering the overheads of the analysis recognising that there will be a need to balance the costs of these indicators with the risk of problematic service usage. Robustness is another aspect, entailing a risk assessment as to the indicator's performance, such as its efficacy (how well the indicator performs, such as its ability to detect situations, positive/negative rates, etc.), representativeness (how definitive or closely the indicator represents actual instances of misuse cf. providing background information), and circumvention, the ways and ease by which the indicator might be bypassed, be it inadvertently or deliberately, e.g. through staggered requests, use of multiple accounts, etc.

The third domain record keeping regards aspects of the audit/ $\log$ data feeding the indicator. The duration dimension refers to the audit data's lifecycle; i.e. whether it processed at run-time (in volatile memory), or is persisted (on disk) and for what duration. This domain also features a sensitivity aspect. The concerns around 
confidentiality, privacy and legal obligations are similar to that in the source information domain, but here they are considered in terms of the data that actually drives the analysis. This reflects that the raw (source) information might exhibit different characteristics to that feeding the indicator, e.g. it may be transformed (e.g. pseudonymised), combined with other data (perhaps increasing sensitivity), stored and batch processed, etc.

The taxonomy, in enumerating these categories, both guides those devising misuse indicators, and enables a more holistic evaluation of the considerations and consequences of such. This is important, as the nature of an indicator has implications regarding costs, overheads, privacy and liability, which we later elaborate. Importantly, note that the taxonomy is intended as a starting point, as a first step serving to frame the development of misuse detection mechanisms. The relevance and applicability of a particular category of concern, and its implications, will of course depend on context. As a living instrument, the taxonomy can be adapted and evolved as new considerations are raised, and according to the specifics of the organisation and service, regulatory guidance, etc.

\subsection{Logging for analysis}

Misuse indicators are driven by information. Therefore implementing indicators entails capturing audit data that reflects particular characteristics and criteria of customer usage to drive subsequent analysis. This involves recording relevant information for supporting the indicator, which can include, e.g., details of the customer (id, IP addresses), transaction (e.g. time, nature of the call), service inputs, resulting outputs, details of intermediate processing, amongst others [26].

Such information is processed as appropriate for the indicator. Again, cloud systems already capture data of customer service interactions, e.g. for billing and system management, and thus might leverage existing methods for monitoring, possibly without significant additional overheads. Analysis may be manual, batch processed, or real time, depending on the particular aims; though many landscaping activities will be manual or periodic.

\section{MOVING FORWARD: INDICATORS IN PRACTICE}

We next use two exemplars to both (i) demonstrate the feasibility and potential for misuse indicators, and (ii) highlight that there are a range of methods for implementing an indicator, each with different implications. To reiterate, the purpose of the indicators is not to definitively uncover misuse (though it might in some instances), but rather to highlight situations warranting consideration. That is, the indicators help direct attention and enable the appropriate follow-on actions.

Naturally there are a vast range of possible indicators; as discussed, misuse concerns are contextual. The following exemplars are instances of general indicators that help providers navigate and explore the 'search space' of service use for possible areas of concern. That is, both exemplars aim to be exploratory, of the sort a provider might employ when attempting to uncover new, or to gain more information of, possible situations of concern. These contrast with those indicators that narrowly look for specific instances (e.g. signatures) of known misuse and seek to be more definitive (see taxonomy representativeness). Naturally, building such specific indicators would require prior knowledge (and data) of what misuse explicitly entails, and as AIaaS is a new area, currently there is little available 'ground truth' or data of definitive of situations of misuse. Therefore, our exemplars consider these more general-type indicators, which, indeed, are representative of the type a provider would initially use to better understand their service usage and for exploring possible situations of concern. Such indicators will play a crucial role in supporting the identification, discovery and mitigation of new and emerging risks of misuse. Moreover, these general methods also serve as as an important first step, as a precursor, to complement the development of more direct, specific and targeted indicators that may be devised in time.

\section{SURVEILLANCE EXEMPLAR}

Our first exemplar indicator considers a usage context suggestive of surveillance. Many of the major AIaaS providers support services that process faces, e.g. facial recognition, emotion detection, etc. Intuitively, face services can be inappropriately used [26].

One possible indicator of potential surveillance is where a facial recognition service is used to process a large number of different faces. This can suggest some form of biometric processing of individuals at scale, possibly indicating inappropriate population surveillance. Such use contrasts with, e.g., a scenario where one continually processes a small number of similar faces, such as for an office's access control system.

In line with this, we now explore an exemplar misuse indicator - where a face service is used to detect many unique faces over time. Note that while many face-related indicators are possible, we use this to illustrate the potential for a particular trait-based indicator and to highlight some of the practical considerations regarding overheads. Note that this indicator (many different faces), isn't definitive of misuse - such processing may well be legitimate. Rather, this broad indicator aims to draw the provider's attention to particular customer behaviours for possible investigation.

Before detailing our exemplar indicator, we first illustrate how the taxonomy helps guide the indicator's design and development. For access level, we consider the nature of what is monitored. Here it is content, and could be the image of the face or its encoding - each with sensitivity implications, both as a source and a record. Here using encodings appears appropriate: they are byproducts of the service itself, and logging encodings (cf. the actual images) reduces overheads and sensitivity concerns, particularly given they will be stored for a period to support analysis (duration). Further, face encodings reduce the overheads of storage, and the indicator itself has computational implications we explore below. The analysis type is trait-based, given there is some focus on a specific type of behaviour. Concerning scale, analysis is customer-specific, as it relates to their service usage. In terms of robustness, we do show reasonable efficacy (face recognition performs well), however, this indicator is meant to be broadly indicative to highlight areas for further exploration, rather than being definitive of misuse (representativeness).

\subsection{Clustering faces}

This indicator analyses the logs representative of a facial recognition service to indicate the number of different faces a customer 
processes. For this, we compare faces in the log for likeness, using clustering methods, to determine the number of different faces seen over a period of time.

6.1.1 Face comparisons. ML face analyses entails face encodings: a vector derived from a model for faces. For two images of the same person, the distance between the encoding vectors will be small; for different individuals, the encodings will be further apart [40]. Because each image varies (lighting, positioning, angle, facial expression, etc.), encodings for two face images generally won't perfectly align (the vectors will differ), but will be close. We use a distance metric to determine whether two images are likely of the same person.

6.1.2 Clustering approaches. One approach for estimating how many different faces a customer processes is to count the number of times a face was submitted to the service. This entails recording the face vector in a log, so the entries can then be clustered and counted - each cluster representing an individual (to some accuracy).

Many clustering approaches require the number of clusters to be specified as a parameter. However, here our aim is to discover the number of clusters (one per individual) of the face encodings. As such, we use DBScan [12] which operates to find the number of clusters within the data, based on a specified distance threshold $(\epsilon)$. $\epsilon$ specifies the max distance between two records to be considered neighbours; i.e. here, by which two face encodings can be considered the same person. We use an experiment (below) to determine an appropriate $\epsilon$ for faces representing the same person.

This surveillance exemplar aims to explore the feasibility and practical considerations of an indicator. As such, we first consider what we term the base approach: applying DBScan clustering over a complete log of face encodings to assess its performance in uncovering the number of different faces contained within. However, given the overheads of that analysis, and recognising that cloud usage logs can rapidly grow, we also explored a periodic approach: rather than running the clustering approach over the entire log of a customer's service interactions, we employ a simple iterative method that maintains a list of the unique face encodings previously observed. Here, DBScan runs periodically (every 20k log entries) over those (new) entries and the list, where the list of unique faces is replaced with the unique encodings from that round of clustering. In undertaking different approaches we seek to highlight that different implementations bring different considerations.

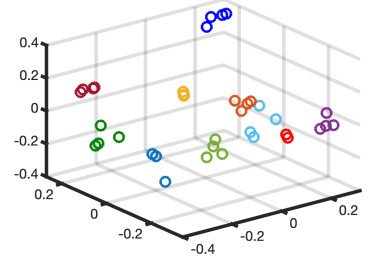

(a)

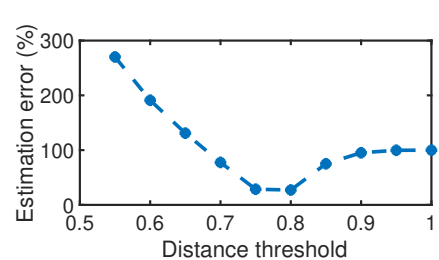

(b)
Figure 1: (a) PCA plot of face encodings, showing each individual's (i.e. colour) encodings closely align. (b) DBScan estimation for different $\epsilon$ (distance threshold).

\subsection{Experimental setup}

To explore (i) the potential for clustering of faces; and (ii) to indicate the associated overheads in doing so, we now outline an experiment leveraging two public datasets: ColorFeret [34] by NIST comprising 11338 faces from 994 people; and LFW [23] which includes 13233 faces from 5749 people. We use ColorFeret to determine the appropriate distance threshold parameter $(\epsilon)$ for DBScan, given it contains a number of images for each individual, and use LFW (many more individuals, but fewer photos of each) to generate the face encoding traces (logs).

We generate log files (representative of service usage) where each entry includes a vector encoding representing a face - i.e. that which would be generated as a byproduct of using an AIaaS face service. Encodings are computed using the TensorFlow implementation of FaceNet [38]. Two types of log are generated: fixed trace uses a specified number (5000) of different faces for each version of the log; while varied trace uses a Zipfian distribution-which can be representative of natural/observed phenomena-to select a varied number of different faces for each log size. Some encodings in the $\log$ are repeated to generate the required number of entries.

The analysis was conducted on a machine with 6 cores (Intel Xeon @2.40GHz) with 2 hardware threads per core (i.e. 12 online CPU threads), 64GB RAM, and $16 \mathrm{MB}$ of L3 cache running Ubuntu 18.04. All experiments were ran at maximum CPU frequency. Each experiment was conducted five times, the results averaged.

6.2.1 Selecting epsilon. Our first step was to find an appropriate epsilon (distance threshold) for our DBScan face clustering experiments. FaceNet could detect and generate encodings for 11284 faces in the ColorFeret dataset (a $0.47 \%$ error, the failures due to extreme angles of the face), with at least one encoding for each person in the dataset. To select $\epsilon$, we give these 11284 encodings as input to DBScan, with incremental $\epsilon$ values and measure the estimation error: the proportion in which the clusters represent the true number of different faces. Based on the results (see Fig. 1(b)), we select $\epsilon=0.8$ which delivered the lowest overall estimation error.

6.2.2 Cluster detection. For each generated trace (log of face encodings), we recorded the number of unique people represented within. We then ran DBScan $(\epsilon=0.8)$ over the trace and measured the estimation error.

As Table 2 presents, we see that the method for uncovering different faces shows promise, given we see that the numbers of clusters returned broadly reflects the number of different faces contained in the dataset. Note as the results for the fixed log indicate, the increase in estimation error is not a function of trace size, but of the number of different faces analysed.

\begin{tabular}{|c||c|c|c||c|c|c|}
\hline \multicolumn{1}{|c|}{ Log Size } & \multicolumn{3}{|c||}{ Varied Traces } & \multicolumn{3}{c|}{ Fixed Traces } \\
\hline & diff faces & clusters & est err \% & diff faces & clusters & est err \% \\
\hline $5 \mathrm{~K}$ & 1149 & 1126 & 2.04 & 5000 & 4520 & 9.6 \\
\hline $50 \mathrm{k}$ & 3834 & 3601 & 6.08 & 5000 & 4520 & 9.6 \\
\hline $100 \mathrm{k}$ & 4732 & 4302 & 9.09 & 5000 & 4520 & 9.6 \\
\hline $150 \mathrm{k}$ & 5179 & 4611 & 10.97 & 5000 & 4520 & 9.6 \\
\hline $200 \mathrm{~K}$ & 5438 & 4748 & 12.69 & 5000 & 4520 & 9.6 \\
\hline
\end{tabular}

Table 2: Input traces and base performance 


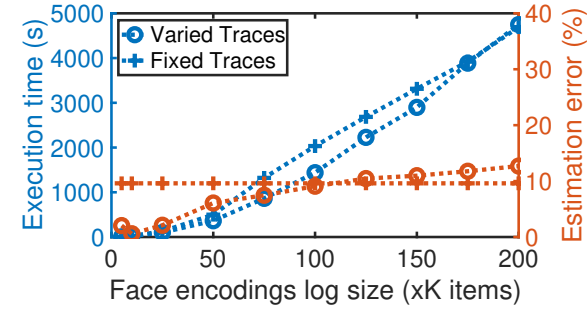

Figure 2: Base performance across varying traces.

6.2.3 Overheads. We next explored the overheads of the approach. Per Fig. 2, we observe the base method delivers a rapidly increasing execution time as the log size grows. We see that the performance implications are similar for both types of logs, confirming the intuition that performance overhead is a function of the number of $\log$ entries. In the context of cloud, which seeks to operate at scale, these overheads bear consideration.

As a means for reducing overheads, we considered the periodic approach (for the varied traces); i.e. where DBScan is run in rounds processing $20 \mathrm{k}$ log encodings, with the unique faces from each round feeding into the processing of the next. In comparison to the base (clustering the entire customer's log), we observe substantial reductions in execution time and memory usage (see Fig. 3).

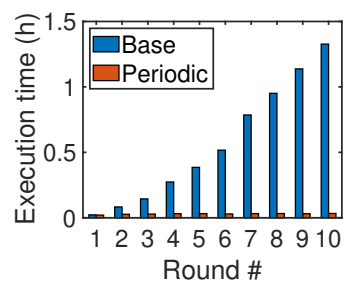

(a) Execution time

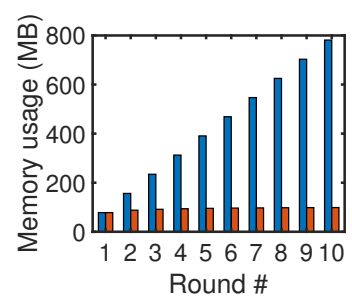

(b) Memory usage
Figure 3: Overheads of the base and periodic methods. Every round $20 \mathrm{~K}$ new face encodings are analyzed.

\subsection{Summary}

The above indicates the potential of looking for particular patterns of behaviours on a defined service; here whether a customer may be profiling many different people, possibly representing population surveillance.

Importantly, that presented represents but one potential indicator, and one implementation towards this. The results confirm that a clustering method (DBScan) performed reasonably well in identifying faces [10] (though given, for example, the increasing error rate, future work might explore the limits of such analysis). And though clustering entails some error, we emphasise that here the purpose is not high accuracy per se, but to provide a heuristic to highlight where further scrutiny is required - i.e. roughly indicating the number of faces being processed may (depending on the context) be sufficient. We also note that the number of faces analysed opens up opportunities for further analysis; e.g. cluster size can have different connotations, raising different concerns: many clusters with few unique faces may indicate e.g. population surveillance, while a small number of clusters (with large number of entries for each) may suggest that individuals are being repeatedly profiled (possibly stalked).

The broader aim of this exemplar is to highlight that considerations are context-dependent. We use our approach to show that implementing indicators is feasible, and at the same time that monitoring brings practical implementation considerations - as shown, more unique faces within the log impacts performance, and overheads depend on the method of analysis. This demonstrates there can be tensions between what is recorded and how it is analysed.

\section{EXEMPLAR: GENERIC LANDSCAPING}

Intuitively, understanding general patterns of behaviour (be they common or different/anomalous) can help inform where attention and investigation is required.

This exemplar aims at such concerns, as it regards uncovering (classes of) customers based on their behaviour. Unlike the previous exemplar that concerned behaviours emblematic of a potentially problematic situation (a type of surveillance), here we consider an even more generic discovery indicator - one that concerns classes of activity, and possible outliers. These types of indicator are to assist providers in focusing effort, where such landscaping can help uncover potential areas warranting attention, including previously unknown issues, so as to inform appropriate responses (be they technical, including developing targeted indicators, or otherwise).

\subsection{Uncovering patterns of usage}

The aim of this indicator is to provide insights regarding the landscape of customer behaviour. Here, we seek to illustrate one way in which the usage space can be explored and dissected.

We focus on discovery-based means of analysing metadata acrosstenants, which raises different (taxonomy) considerations to the previous surveillance exemplar. We again explore the use of clustering methods to group customer usage patterns and highlight common behaviours and those anomalous. These clusters represent groups of customers who exhibit similar and particular behaviour patterns (which may, for example, reflect particular application types), and can help guide a provider on where to focus attention.

We illustrate this using two common clustering approaches, KMeans [29] and Gaussian Mixture Model (GMM) [35]. Both group their observations into a specified number of clusters. This can be adjusted to be more granular or inclusive, giving providers the flexibility to navigate the usage landscape.

\subsection{Experimental setup}

For this study, it is useful to examine a dataset reflecting real customer behaviour. Despite a lack of publicly available AIaaS usage logs, recently a dataset was released containing the real-world customer metadata from 14 days use of Microsoft's Azure Function as a Service (FaaS) [42]. Similar to AIaaS, FaaS also entails invoking particular functions via a web API, and thus is analogous to AIaaS service invocations. In this way, the Azure data is suitable for the purposes here of demonstrating the potential for mapping a usage landscape. The dataset includes the function invocation count (FIC) 


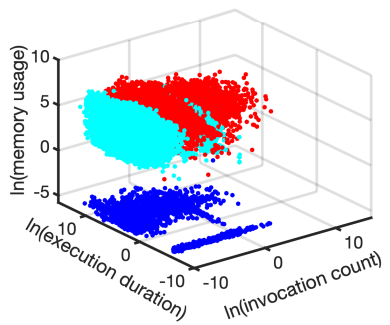

(a) $k=3$

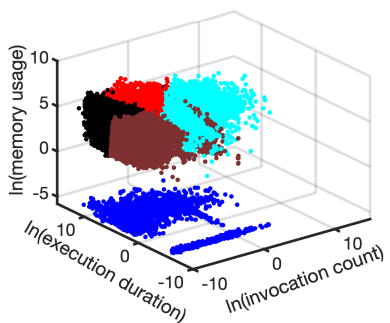

(b) $k=5$

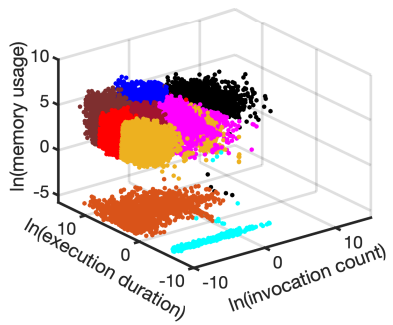

(c) $k=9$

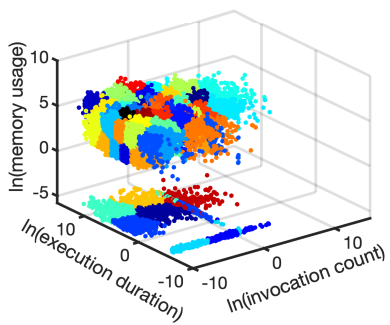

(d) $n c=50$

Figure 4: KMeans clustered scatter plots. $k$ stands for number of clusters.

\begin{tabular}{|l|l|}
\hline $\mathbf{k}$ & Cluster sizes \\
\hline 2 & $\{41264,31095\}$ \\
\hline 3 & $\{39983,29747,2629\}$ \\
\hline 5 & $\{20131,18554,17918,13127,2629\}$ \\
\hline 9 & $\{13483,11822,11529,9528,9230,9142,4998,1854,773\}$ \\
\hline 50 & $\{3692,3201,2613,2600,2581, \ldots, 421,324,272,237,192\}$ \\
\hline
\end{tabular}

Table 3: KMeans cluster sizes for the Azure data trace.

per minute, and the function execution duration (FED) and application memory allocation (AMA) data averaged and with percentiles per 24 hours.

From the Azure data we derive two datasets. The first represents the normalised FIC, FED and AMA at minute-level granularity over the period of seven days. As FED and AMA are only provided for every 24 hours, we use the provided percentiles (indicating the probability distribution) to regenerate representative minute-granularity FED and AMA data. As the AMA is only available per application (each application is associated with one or more function calls), we assign the memory usage value uniformly across the application's functions. To provide another landscaping illustration, the second dataset more narrowly encapsulates invocation details, including the normalised hourly summaries of FIC (mean for each hour) and FED (derived from the percentiles) over 14 days.

In both cases, we have a dataset of 72359 unique entries, which here can be considered representative of the behaviour of $\sim 72 \mathrm{k}$ customers interacting with the provider. Values were log normalised for analysis, and conducted on the same hardware earlier described.

\subsection{Results of usage record clustering}

We first used KMeans to explore the more detailed (first) dataset as described above. Fig. 4 and Table 3 show the results of the KMeans for different numbers of clusters $(k)$. We observe that there are several large clusters, showing more common patterns of behaviour, as well as a number of smaller clusters. This information can support providers in understanding the service usage landscape, e.g., to identify common classes of behaviour as well as those behaviours that are less frequent. We see that increasing $k$ effectively works as a means to drill-down on particular behaviours. The ability to adjust sensitivity $(k)$ aids discovery and landscaping processes, enabling more granular or macro-oriented analyses as and when appropriate.
The above gives a general view over how a landscape can be explored. We also conducted a similar experiment on the more narrow, function-oriented (second) dataset, using GMM to illustrate a different approach. For want of space, we do not present fuller details - however, for a specified five clusters, the resulting cluster sizes were: $\{51120,18902,2318,16,3\}$. Interesting here is that we observe some large clusters, as well as two very small clusters with a size of 16 and 3 (which stand out by way of their large FIC and FEDs). This is informative, revealing outlier and anomalous behaviour patterns that may warrant further investigation.

\subsection{Summary}

Our results show the propensity for clustering methods for helping to reveal patterns of behaviour, including those general and common (i.e. large clusters), as well as identifying outliers and anomalies. Both might be interesting from a monitoring perspective, in helping providers better understand how their services are commonly used, while identifying outliers and anomalous customer behaviours that may require investigation. Moreover, methods that allow tuning and tailoring, whereby parameters effectively allow a zooming in/out, can be useful by enabling a more flexible analysis.

To reiterate, here the aim is simply to highlight the potential for discovery-oriented indicators, and one approach for such. In practice, various different approaches can be taken to explore the landscape. Moreover, there are a wide range data points available to a provider that could be considered as part of any such analyses (e.g. more granular data points, disk or service $\mathrm{I} / \mathrm{O}$, etc).

\section{DISCUSSION}

As AIaaS grows in prominence, so too does the risk of AI services being used problematically. Indeed, providers have a large and growing customer base, making it both increasingly challenging, and important, that their offerings aren't undermined through misuse, and that their obligations and responsibilities are met.

As we have argued, there is a need for technical measures that enable the monitoring of AIaaS (working to help uncover misuse), and that the providers themselves are well-placed to implement such. In arguing this need for more oversight of customer behaviour, we have also outlined various considerations for doing so.

Note, however, that AIaaS misuse concerns are contextual - the extent to which monitoring takes place, and the various approaches employed, can differ according to the provider, customers, service type, applicable regulation, and other aspects. Our contributions 
are therefore not to show specific examples of misuse, but rather, to highlight the importance and potential for technical means that help uncover such. Our taxonomy offers an initial framework of relevant monitoring considerations, and our landscaping indicator exemplars indicate viability and are representative of how monitoring might operate. In this way, our paper represents a tangible first step, bringing attention to this area, and paving a practical way forward.

Limitations: Naturally, in presenting a path forward, our work is subject to some limitations. First, as AIaaS is nascent, there is a lack of data regarding AIaaS usage, let alone specific instances of misuse. This is unsurprising given AIaaS misuse is an area little discussed. That said, we did explore the Azure FaaS usage data, which is realworld and analogous with AIaaS. While sufficient for demonstrating the exploration of a usage landscape, actual AIaaS data, particularly that regarding real examples of problematic situations, would assist in exploring indicators more definitive of misuse.

Moreover, as a new area, there are understandably few widelyknown AIaaS examples that clearly represent misuse. Though the problem of AIaaS misuse is intuitive-and the restrictions on law enforcement's use of face services and the directions emerging regulatory regimes both show that the concerns are real-details of other scenarios (particularly those more technically complex) are yet to emerge.

These aspects may be addressed as AIaaS matures, its adoption increases, and more awareness is brought to AIaaS misuse - one key aim of this paper.

In light of the above, our exemplars have therefore sought to illustrate particular considerations, demonstrating the concept and its feasibility. Specifically, they show how the usage landscape may be surveyed to explore misuse. The surveillance exemplar broadly represents a general usage pattern that has potential to be problematic, while the more generic exemplar shows how a provider might landscape and 'hone-in' on patterns of interest. These types of indicators are important and necessary for understanding the nature of service usage, providing the means for discovering situations of concern. That is, they help providers gain the knowledge and data for determining 'known' patterns of misuse, from which narrow, specific indicators, more definitive of misuse can be developed.

Note also that the issues arising from AI service usage are inherently socio-technical. Though we have focused on the technical aspect, our work is intended to complement other measures for tackling such concerns, be they organisational, legal, economic, social, and so on.

We next outline a selection of considerations representing opportunities for discussion and future research.

\subsection{Operational considerations}

First, we explore a number of operational considerations regarding AIaaS misuse detection.

Balancing monitoring costs: Monitoring service customers comes with cost. This includes the cost of developing and implementing such measures (e.g. staff and processes), possible legal/compliance considerations [8], and the performance (resource) implications of monitoring - from collecting and storing log data, to, as we have outlined, its analysis. However, these shouldn't be thought of as trade-offs, but rather a balance - there will be requirements for monitoring and there will be risks and other aspects requiring consideration. And what is appropriate will depend on the specific circumstances, aspects of which our taxonomy captures. From a performance perspective, there is scope for work optimising the implementation of various indicators. This could involve potentially leveraging the fact that providers already undertake logging and analysis, e.g., for billing, security, maintenance, and for methods that advantage the nature of cloud, e.g. perhaps operating during periods of low service usage.

Data management and obligations: Data for analysis might contain confidential, private or sensitive data. This brings responsibilities; perhaps legal, e.g. contractual or data protection-oriented (for personal data), or commercial, e.g. where leaking such information could have business repercussions. Therefore means for appropriately storing, managing, and accessing data will be needed, as will any broader implications (legal or otherwise) of how monitoring operates [8]. What data is appropriate for use for misuse monitoring, when, and how it is managed depends on context. Not all transactional data will have the same concerns (e.g. request metadata is often arguably less risky than request content) - as the sensitivity dimensions of our taxonomy reflect. Note that providers do already have experience in recording data (e.g. for billing, maintenance), and should have considered these sorts of issues to some extent.

Evasion: Important is considering the capacity for which an indicator might be evaded, as encapsulated by circumvention in our taxonomy. This might involve a customer changing behaviour, e.g. by obfuscating their operations, by way of extra transactions, timing their requests, or using multiple accounts to diminish their visibility. This can result in a 'cat-and-mouse' scenario, where adversaries work to avoid detection, and platforms adapt to these changes - as we see in other domains. This, however, reinforces the need for the general, landscaping indicators-of the type our exemplars reflect-to inform providers of new forms of misuse, patterns of behaviours reflecting evasion, and so on.

Moreover, cloud represents paid-for corporate services, which is a different context to issues that arise, for example, through 'anonymous' social media accounts. This simplifies identifying and dealing with customers, and may possibly deter and hinder adversarial behaviours. In all, there are clear areas for research in this space.

\subsection{Platform considerations}

Incentives: Worth exploring are the incentives for providers to undertake misuse monitoring. At first glance, there appear incentives for not acting, and turning a blind eye. This may be so that providers continue to profit, and because uprooting controversial applications that were previously operating unhindered may itself generate criticism. And having knowledge of improper behaviour can potentially come with pressure to act. One business consideration is whether customers would accept having their service usage actively monitored [26]; though note the provider is already processing their data by their use of the service, and already undertakes monitoring as part of supplying that service.

That said, there are consequences of maintaining ignorance or not taking action. We have discussed the reputational implications 
for providers supporting untoward applications, and there may be legal incentives, existing and emerging, obliging providers to be more responsible for their offerings [8].

In short, service providers will be unlikely to continue to simply wash their hands of what happens via their platforms. Though we do not here explore the legal and business dimensions to 'AIaaS' (see [8] for some discussion of such), we highlight there are a wealth of research opportunities in the space.

The power of platforms: AlaaS providers are the 'tech giants', with the data, expertise, compute and other resources to provide the state of the art AI functionality. As such, many will come to rely on AIaaS for their applications, as the services offer a capability otherwise beyond one's reach.

This places AIaaS providers in a powerful position. One issue is gatekeeping, where providers determine who may or may not have access. Who decides what is appropriate is important - the providers' commercial interests may be very different from broader societal concerns. Given current regulatory directions, there appears both scope and appetite for oversight bodies to set directions regarding monitoring, misuse, and what actions are acceptable, as well as the role for other stakeholders, e.g. regulators, industry, civil society. Transparency and accountability regimes are needed that enable the actions of providers (e.g. monitoring processes, removing access) to be effectively challenged. The specifics regarding external oversight and scrutiny over the use, reliance, monitoring and actions of cloud services is an area requiring consideration.

Legal uncertainties We have described that emerging law and regulation will likely oblige providers to be more responsible for their services. Currently, however, there are legal implications regarding a platform's monitoring for misuse. For instance, where the data being analysed for monitoring is personal and thus falls within data protection law, its processing for monitoring will not be lawful unless one of several conditions specified in law is met (e.g., see GDPR [15] Arts. 6 and 9). These bases are limited, and may be difficult for providers to rely on - as one example, it would be challenging for a provider to obtain explicit consent from each individual whose data might be encapsulated in the vast range and volume of AIaaS service requests. Of course, the issues depend on the circumstances (see [8] for detailed discussion). How the roles, responsibilities, and possible tensions between competing concerns (e.g. privacy vs service misuse) are balanced in an AIaaS context are areas requiring consideration.

In sum, AIaaS misuse monitoring is an important area that warrants attention, raising opportunities for research and other interventions from across various domains.

\section{CONCLUDING REMARKS}

There is a growing public discourse calling for more responsible and accountable AI. Given AI services will play an increasing role in supporting applications, we have argued the potential for monitoring AIaaS usage in identifying and indicating situations of possible concern. In elaborating the concept of misuse indicators, providing a taxonomy describing relevant considerations, and presenting exemplar indicators, we have shown the feasibility and potential for misuse indicators, and highlighted various considerations and their practical implications.
What we present is a way forward on this important topic. Our broader aim is to draw attention to issues of AIaaS, and the practical considerations and opportunities in the area, to help work towards ensuring that AI services (including their providers and users) operate in manner that better aligns with societal interests.

\section{ACKNOWLEDGEMENTS}

We acknowledge the financial support of the UK EPSRC (EP/P024394/1, EP/R033501/1) and Microsoft via the Microsoft Cloud Computing Research Centre.

\section{REFERENCES}

[1] Mohiuddin Ahmed, Abdun Naser Mahmood, and Md Rafiqul Islam. 2016. A Survey of Anomaly Detection Techniques in Financial Domain. Future Generation Computer Systems 55 (2016), 278-288.

[2] Amazon. 2019. Build an AI-driven Application. https://aws.amazon.com/ machine-learning/ai-services/

[3] Amazon. 2019. DetectFaces. https://docs.aws.amazon.com/en_pv/rekognition/ latest/dg/API_DetectFaces.html

[4] Amazon. 2020. Amazon Rekognition FAQs. https://aws.amazon.com/ rekognition/faqs/?nc $=$ sn\&loc $=7$

[5] Ho Bae, Jaehee Jang, Dahuin Jung, Hyemi Jang, Heonseok Ha, and Sungroh Yoon. 2018. Security and Privacy Issues in Deep Learning. arXiv preprint arXiv:1807.11655 (2018).

[6] Elettra Bietti. 2020. From Ethics Washing to Ethics Bashing: A View on Tech Ethics from within Moral Philosophy. In Proceedings of the ACM Conference on Fairness, Accountability, and Transparency (FAT ${ }^{*}$. ACM, 210-219. https: //doi.org/10.1145/3351095.3372860

[7] Miles Brundage, Shahar Avin, Jack Clark, Helen Toner, Peter Eckersley, Ben Garfinkel, Allan Dafoe, Paul Scharre, Thomas Zeitzoff, Bobby Filar, et al. 2018. The Malicious Use of Artificial Intelligence: Forecasting, Prevention, and Mitigation. arXiv preprint arXiv:1802.07228 (2018).

[8] Jennifer Cobbe and Jatinder Singh. 2021. Artificial Intelligence as a Service: Legal Responsibilities, Liabilities, and Policy Challenges. Available on SSRN. https://papers.ssrn.com/sol3/papers.cfm?abstract_id=3824736

[9] Rodrigo da Rosa Righi, Matheus Lehmann, Marcio Miguel Gomes, Jeferson Campos Nobre, Cristiano André da Costa, Sandro José Rigo, Marcio Lena, Rodrigo Fraga Mohr, and Luiz Ricardo Bertoldi de Oliveira. 2019. A Survey on Global Management View: Toward Combining System Monitoring, Resource Management, and Load Prediction. Journal of Grid Computing 17, 3 (2019), 473-502.

[10] Nameirakpam Dhanachandra and Yambem Jina Chanu. 2017. A Survey on Image Segmentation Methods Using Clustering Techniques. European Journal of Engineering Research and Science 2, 1 (2017), 15-20.

[11] Pedro Domingos. 2012. A Few Useful Things to Know about Machine Learning. Communications of the ACM 55, 10 (2012), 78-87. https://doi.org/10.1145/ 2347736.2347755

[12] Martin Ester, Hans-Peter Kriegel, Jörg Sander, Xiaowei Xu, et al. 1996. A Density-based Algorithm for Discovering Clusters in Large Spatial Databases with Noise. In Proceedings of the 2nd ACM SIGKDD International Conference on Knowledge Discovery and Data Mining, Vol. 96. 226-231.

[13] EU Commission. 2020. Digital Services Act. https://ec.europa.eu/digital-singlemarket/en/digital-services-act-package

[14] EU Commission. 2021. Proposal for a Regulation of the European Parliament and of the Council Laying Down Harmonised Rules on Artificial Intelligence (Artificial Intelligence Act) and Amending Certain Union Legislative Acts. $\underline{\operatorname{COM}(2021) 206}$ (2021).

[15] European Union. 2016. Regulation (EU) 2016/679 of the European Parliament and of the Council of 27 April 2016 on the protection of natural persons with regard to the processing of personal data and on the free movement of such data, and repealing Directive 95/46/EC (General Data Protection Regulation).

[16] Gilberto Fernandes, Joel JPC Rodrigues, Luiz Fernando Carvalho, Jalal F AlMuhtadi, and Mario Lemes Proença. 2019. A Comprehensive Survey on Network Anomaly Detection. Telecommunication Systems 70, 3 (2019), 447-489.

[17] Google. 2019. AI and Machine Learning Products. https://cloud.google.com/ products/ai/building-blocks/

[18] Google. 2019. Our Approach to Facial Recognition. https://ai.google/ responsibilities/facial-recognition/

[19] Samuel Greengard. 2019. Will Deepfakes Do Deep Damage? Communications of the ACM 63, 1 (Dec. 2019), 17--19. https: //doi.org/10.1145/3371409

[20] Kori Hale. 2020. Amazon Halts Police of its Facial Recognition Technology Following Years of Criticism. https://www.forbes.com/sites/korihale/2020/ 
06/15/amazon-microsoft--ibm-slightly-social-distancing-from-the-8-billionfacial-recognition-market

[21] Douglas Harris. 2018. Deepfakes: False Pornography Is Here and the Law Cannot Protect You. Duke Law and Technology Review 17 (2018), 99.

[22] K. Hazelwood, S. Bird, D. Brooks, S. Chintala, U. Diril, D. Dzhulgakov, M. Fawzy, B. Jia, Y. Jia, A. Kalro, J. Law, K. Lee, J. Lu, P. Noordhuis, M. Smelyanskiy, L. Xiong, and X. Wang. 2018. Applied Machine Learning at Facebook: A Datacenter Infrastructure Perspective. In Proceedings of 2018 IEEE International Symposium on High Performance Computer Architecture. 620-629.

[23] Gary B Huang, Marwan Mattar, Tamara Berg, and Eric Learned-Miller. 2008 Labeled Faces in the Wild: A Database for Studying Face Recognition in Unconstrained Environments. In Workshop on Faces in Real-Life Images: Detection, Alignment, and Recognition.

[24] IBM. 2019. IBM Watson Products and Solutions. https://www.ibm.com/watson/ products-services

[25] Gareth James, Daniela Witten, Trevor Hastie, and Robert Tibshirani. 2013 An Introduction to Statistical Learning. Vol. 112. Springer.

[26] Seyyed Ahmad Javadi, Richard Cloete, Jennifer Cobbe, Michelle Seng Ah Lee, and Jatinder Singh. 2020. Monitoring Misuse for Accountable 'Artificial Intelligence as a Service'. In Proceedings of the AAAI/ACM Conference on AI, Ethics, and Society. ACM, New York, NY, USA, 300-306.

[27] Michael Kwet. 2020. The Real Question: When Do We Ban Human Drivers? https://theintercept.com/2020/07/14/microsoft-police-state-mass-surveillancefacial-recognition/

[28] Hung-Jen Liao, Chun-Hung Richard Lin, Ying-Chih Lin, and Kuang-Yuan Tung 2013. Intrusion Detection System: A Comprehensive Review. Journal of Network and Computer Applications 36, 1 (2013), 16-24.

[29] Stuart Lloyd. 1982. Least Squares Quantization in PCM. IEEE Transactions on Information Theory 28, 2 (1982), 129-137.

[30] Microsoft. 2019. Cognitive Services. https://azure.microsoft.com/en-gb/services/ cognitive-services

[31] Microsoft. 2019. Cognitive Services Pricing - Face API. https://azure.microsoft. $\mathrm{com} / \mathrm{en}$ - gb/pricing/details/cognitive-services/face-api/

[32] Sharon Naker and Dov Greenbaum. 2017. Now You See Me: Now You Still Do: Facial Recognition Technology and The Growing Lack of Privacy. BUJ Sci. \& Tech. L. 23 (2017), 88-122.

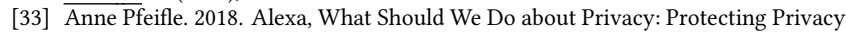
for Users of Voice-Activated Devices. Wash. L. Rev. 93 (2018), 421-458.

[34] P Jonathon Phillips, Harry Wechsler, Jeffery Huang, and Patrick J Rauss. 1998 The FERET Database and Evaluation Procedure for Face-recognition Algorithms. Image and vision computing 16, 5 (1998), 295-306.

[35] Douglas A Reynolds. 2009. Gaussian Mixture Models. Encyclopedia of biometrics 741 (2009)

[36] M. Ribeiro, K. Grolinger, and M. A. M. Capretz. 2015. MLaaS: Machine Learning as a Service. In Proceedings of IEEE 14th International Conference on Machine
Learning and Application. 896-902.

[37] Francesca Rossi. 2019. Building Trust in Artificial Intelligence. Journal of international affairs 72, 1 (2019), 127-134

[38] David Sandberg. 2020. Face Recognition Using Tensorflow. https://github.com/ davidsandberg/facenet

[39] Daniel Schiff, Justin Biddle, Jason Borenstein, and Kelly Laas. 2020. What's Next for AI Ethics, Policy, and Governance? A Global Overview. In Proceedings of the AAAI/ACM Conference on AI, Ethics, and Society. 153-158. https://doi.org/10. 1145/3375627.3375804

[40] F. Schroff, D. Kalenichenko, and J. Philbin. 2015. FaceNet: A Unified Embedding for Face Recognition and Clustering. In Proceedings of the Conference on Computer Vision and Pattern Recognition. 815-823.

[41] Andrew D. Selbst, Danah Boyd, Sorelle A. Friedler, Suresh Venkatasubramanian, and Janet Vertesi. 2019. Fairness and Abstraction in Sociotechnical Systems. In Proceedings of the Conference on Fairness, Accountability, and Transparency (Atlanta, GA, USA) (FAT* '19). Association for Computing Machinery, New York, NY, USA, 59?68. https://doi.org/10.1145/3287560.3287598

[42] Mohammad Shahrad, Rodrigo Fonseca, Íñigo Goiri, Gohar Chaudhry, Paul Batum, Jason Cooke, Eduardo Laureano, Colby Tresness, Mark Russinovich, and Ricardo Bianchini. 2020. Serverless in the wild: Characterizing and optimizing the serverless workload at a large cloud provider. In 2020 USENIX Annual Technical Conference (ATC)). 205-218.

[43] The Partnership on AI. 2020. About Us. https://www.partnershiponai.org/about/

44] The Partnership on AI. 2020. When AI Systems Fail: Introducing the AI Incident Database. https://www.partnershiponai.org/aiincidentdatabase/

[45] The Royal Society. 2018. The Impact of Artificial Intelligence on Work. https://royalsociety.org/-/media/policy/projects/ai-and-work/evidencesynthesis-the-impact-of-AI-on-work.PDF

[46] Cristian Vaccari and Andrew Chadwick. 2020. Deepfakes and Disinformation: Exploring the Impact of Synthetic Political Video on Deception, Uncertainty, and Trust in News. Social Media + Society 6, 1 (2020), 1-13. https://doi.org/10.1177/ 2056305120903408

[47] Emily West. 2019. Amazon: Surveillance as a Service. Surveillance \& Society 17, $1 / 2(2019), 27-33$

[48] Maranke Wieringa. 2020. What to Account for When Accounting for Algorithms: A Systematic Literature Review on Algorithmic Accountability. In Proceedings of the ACM Conference on Fairness, Accountability, and Transparency $\left(\right.$ FAT $\left.^{*}\right)$. ACM, 1-18. https://doi.org/10.1145/3351095.3372833

[49] Yi Zeng, Enmeng Lu, Yinqian Sun, and Ruochen Tian. 2019. Responsible Facial Recognition and Beyond. arXiv:1909.12935 (2019).

[50] Remco Zwetsloot, Roxanne Heston, and Zachary Arnold. 2019. Strengthening the US AI Workforce. Center for Security and Emerging Technology, Washington $\underline{\mathrm{DC}}(2019)$ 